\title{
Liver Resection for Metastasis of Rectal Cancer Origin in a Polycystic Liver
}

\author{
Mircea Pavel ${ }^{1}$, Alexandru Procop ${ }^{2}$, Patricia Boeti-Sarbu ${ }^{1,3}$, Radu Dumitru $^{3,4}$, Traian Dumitrascu ${ }^{1,3}$
}

${ }^{1}$ Center of General Surgery and Liver Transplant, Fundeni Clinical Institute, Bucharest, Romania 2Department of Pathology, Fundeni Clinical Institute, Bucharest, Romania

3"Carol Davila" University of Medicine and Pharmacy, Bucharest, Romania

${ }^{4}$ Department of Medical Imaging and Interventional Radiology, Fundeni Clinical Institute, Bucharest, Romania

\section{ABSTRACT}

Liver metastases of colorectal origin are a common pathology, and the most frequent indication for liver resection. Associated chronic liver pathology may potentially increase the difficulty of a liver resection. Hereby, it is presented the case of a 67-year-old woman with a liver metastasis of rectal cancer origin with concomitant polycystic liver disease. An atypical liver resection was performed with intraoperative ultrasound guidance. Liver tumors developed on a polycystic liver are rarely described. For these particular cases, the diagnosis is challenging, but liver resection can be safely performed with the intraoperative ultrasound guidance.

Key words: liver resection; metastasis; colorectal cancer; polycystic liver disease

\section{INTRODUCTION}

Liver metastases of colorectal origin are a common pathology, and the most frequent indication for liver resection (1). In the last years, it was observed an increased incidence of liver resections for colorectal liver metastases (1). In highvolume centers, the postoperative morbidity and mortality rates after liver resection for colorectal liver metastases are relatively low (2). Neoadjuvant chemotherapy has been suggested for colorectal liver metastases, and it was proven to be of benefit, particularly for those metastases initially deemed unresectable (3). Nevertheless, a patient with colorectal liver metastases should be managed in a multidisciplinary team, and the approach should be tailored to each patient(4).

The type of liver resection for colorectal liver metastases has changed over the time from standard hepatectomies to parenchyma-sparing liver resections (1). Associated chronic liver pathology may potentially increase the difficulty of a liver resection.

Hereby, it is presented the case of a patient with a liver metastasis of rectal cancer origin with concomitant polycystic liver disease.
Corresponding author:

Traian Dumitrascu, MD

"Carol Davila" University of Medicine and Pharmacy, Center of General Surgery and Liver Transplant Fundeni Clinical Institute Fundeni Street no 258, 022328 Bucharest, Romania E-mail: traian.dumitrascu@umfcd.ro
Received: 10.03.2018 Accepted: 13.04 .2018 


\section{CASE PRESENTATION}

A 67-year-old woman was referred to our Department for further investigation of a liver mass diagnosed at abdominal ultrasound examination. Her medical history included polycystic liver disease, and previous surgery in 2015 (i.e., low anterior colorectal resection with stapled anastomosis) for $\mathrm{pT} 3 \mathrm{pN} 1 \mathrm{cMO}$ adenocarcinoma of the mid rectum, with neoadjuvant radiotherapy and adjuvant chemotherapy (FOLFOX regimen). During the follow-up, the patient was discovered with a liver mass. Contrast-enhanced computed tomography (fig. 1) and magnetic resonance imaging (fig. 2) revealed a single metastasis of segments IV-VIII of the liver, measuring $70 \mathrm{~mm} / 65 \mathrm{~mm} /$ $55 \mathrm{~mm}$, and polycystic liver disease. No other distant metastases were detected. Colonoscopy did not reveal any abnormalities. Bioumoral tests were normal, except for elevated alpha-fetoprotein $(9.7 \mathrm{ng} / \mathrm{ml}$; range, $1.3-8 \mathrm{ng} / \mathrm{ml})$, CA $19-9(53.8 \mathrm{Ul} / \mathrm{ml}$; range, 1.2 - $37 \mathrm{UI} / \mathrm{ml}$ ) and carcinoembryonic antigen $(25.8 \mathrm{ng} /$ $\mathrm{ml}$; range, $0.5-7 \mathrm{ng} / \mathrm{ml}$ ).

The patient underwent surgery in June 2017. Intraoperatively, it was confirmed the polycystic liver disease, and a single metastasis of segments IV-VIII of the liver (fig. 3). The intraoperative ultrasound examination was performed to rule out other potential liver metastases, and to guide the liver resection for identified metastasis. On notice, although the liver metastasis was identified at liver palpation, however, its limits were unclear, and it was very difficult to distinguish the margins of metastasis from the surrounding liver cysts (fig. 3). Thus, the intraoperative ultrasound guidance was of utmost importance, and an atypical liver resection of segments IV-VIII was performed. The postoperative outcome was uneventful, except for a minor bleeding/ hematoma revealed on the drainage tube, that was spontaneously solved, and the patient was discharge on postoperative day 12. A white-grayish tumor was observed at gross pathology examination of the operative specimen (fig. 4), and the histological finding was compatible with a metastatic tubulopapillar, well-differentiated adenocarcinoma of colorectal origin.

\section{DISCUSSION}

The liver is considered the most common site for metastatic colorectal cancer (5). Interestingly, it appears that patients with chronically diseased livers have lower

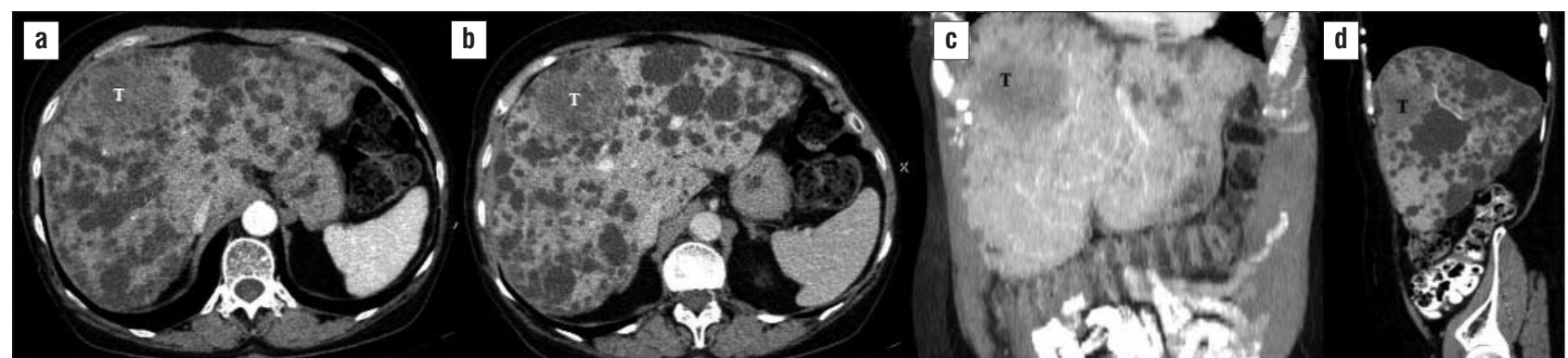

Figure 1 - Contrast-enhanced computed tomography (a) axial plane, arterial phase; (b) axial plane, portal phase; (c) frontal plane, portal phase; (d) sagittal plane, arterial phase showing a hypodense metastasis of segments IV-VIII of the liver (T) and numerous small to medium sized cysts in all liver segments, highly suggestive for the diagnosis of polycystic liver disease

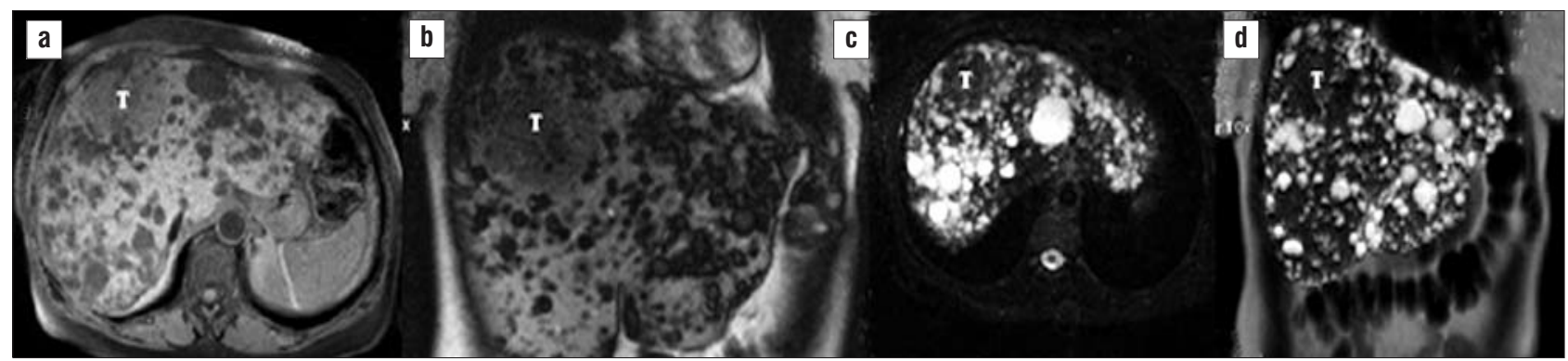

Figure 2 - Magnetic resonance imaging (a) axial plane, T1-weighted; (b) frontal plane, T1-weighted; (c) axial plane, T2-weighted;

(d) frontal plane, T2-weighted showing a hypodense metastasis of segments IV-VIII of the liver (T) and numerous nonenhancing in T1-weighted and hyperintense in T2-weighted liver cysts, highly suggestive for the diagnosis of polycystic liver disease 


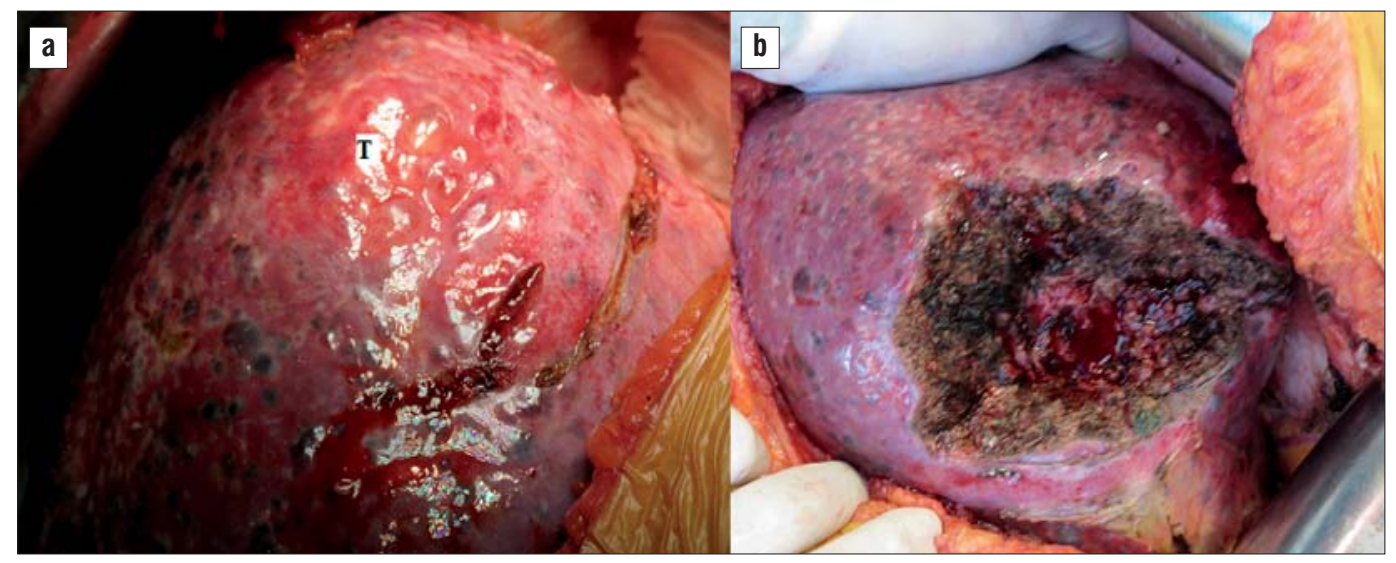

Figure 3 - Intraoperative aspects showing (a) a liver metastasis of segments IV-VIII of the liver (T) and multiple liver cysts, and (b) liver after atypical hepatic resection of segments IV-VIII

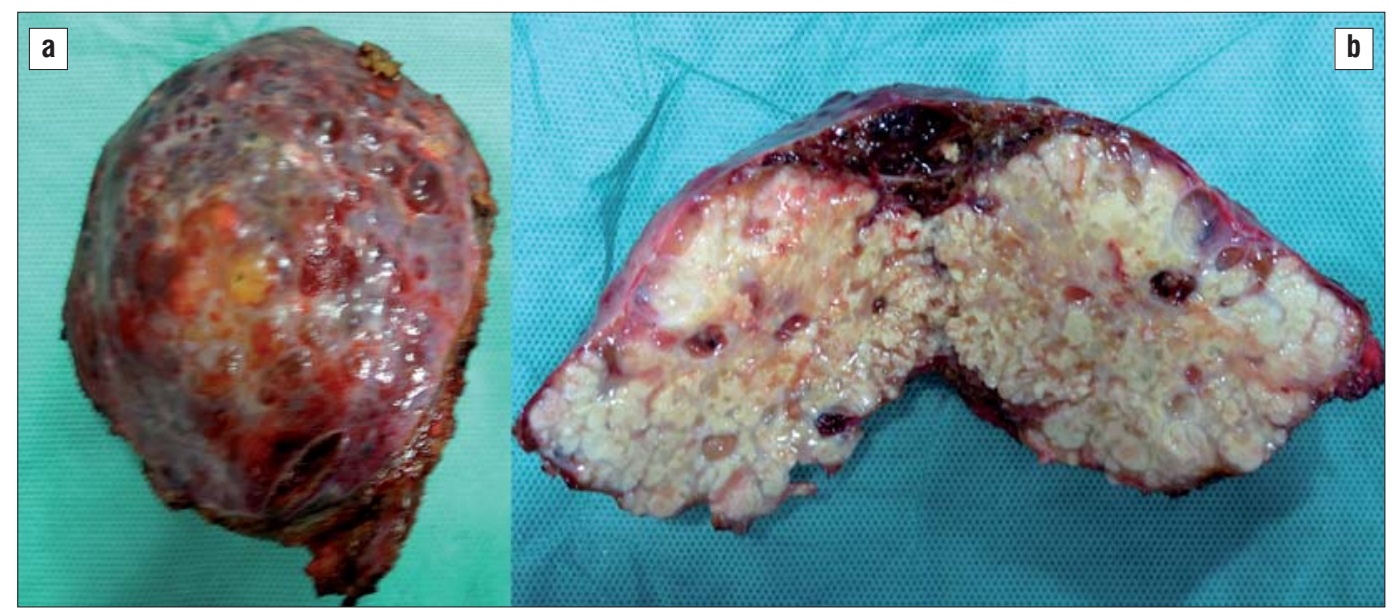

Figure 4 - Gross pathology appearance of the operative specimen showing (a) an atypical resection of segments IV-VIII of the liver, and (b) a white-grayish liver metastasis of rectal adenocarcinoma origin

incidence of colorectal liver metastases $(5,6)$. Thus, low incidence of colorectal liver metastases were previously described in patients with chronic hepatitis $B$ and $C$ virus infection or cirrhosis $(5,6)$.

Polycystic liver disease is an autosomal dominant inherited disease characterized by multiple cysts inside the liver parenchyma, usually more than 20 cysts $(7,8)$. It can occur with or without associated polycystic kidney disease $(7,8)$. The symptoms are mainly related to mass effect of the volume of hepatic cyst, and, rarely, the disease progresses to liver failure or portal hypertension $(7,8)$. The most common imaging methods to diagnose polycystic liver disease are computed tomography and magnetic resonance $(7,8)$.

Most patients with polycystic liver disease do not need any treatment $(7,8)$. However, in few patients, liver resection or transplantation is required $(7,8)$.

Liver resection on a polycystic liver disease could be challenging. Liver resections for hepatic tumors developed on a polycystic liver disease were previously described in only few patients with haemangiomas (9), primary leiomyosarcomas (10), and angiomyxomas (11). Furthermore, other few cases of non-resected liver tumors developed on polycystic liver disease were also described in patients with neuroendocrine tumours (12) or Schneiderian metastatic carcinomas (13). It is worth to mention that liver function tests are usually normal in patients with polycystic liver disease (8). Nevertheless, liver resection on polycystic liver disease can be performed with acceptable morbidity and mortality rates (14).

Noteworthy, data about metastases developed on a polycystic liver disease are scarce (15).

Nowadays, parenchyma-preserving liver resections represent the first choice in surgery for colorectal liver metastases(2). The intraoperative ultrasound examination is very useful to detect some other metastases that were not identified with preoperative imaging work-up 
(16). Contrast-enhanced intraoperative ultrasound examination may potentially increase accuracy to detect other metastases (17). The introperative ultrasound examination not only provides improved diagnosis but also liver resection guidance, with low morbidity and mortality rates (16). In the abovementioned patient, the intraoperative ultrasound examination was mandatory to identify the margins of liver metastasis and thus, to guide liver resection with safe margins.

\section{CONCLUSION}

Liver tumors developed on a polycystic liver are rarely described. For these particular cases, the diagnosis is challenging, but liver resection can be safely performed with the intraoperative ultrasound guidance.

\section{REFERENCES}

1. Botea F, lonescu M, Brasoveanu V, Hrehoret D, Alexandrescu S, Grigorie M, et al. Liver Resections in a High-Volume Center: Form Standard Procedures to Extreme Surgery and Ultrasound-guided Resections. Chirurgia (Bucur) 2017;112(3):259-77.

2. Alexandrescu S, Diaconescu A, lonel Z, Zlate C, Grigorie R, Hrehoret $D$, et al. Comparative Analysis between Simultaneous Resection and Staged Resection for Synchronous Colorectal Liver Metastases - A Single Center Experience on 300 Consecutive Patients. Chirurgia (Bucur) 2017;112(3):278-88.

3. Popescu I, Alexandrescu ST. Surgical options for initially unresectable colorectal liver metastases. HPB Surg 2012;2012:454026.

4. Popescu I, Alexandrescu S. [Hepatic metastasis of colorectal cancer--current therapeutic possibilities]. Chirurgia (Bucur) 2010; 105(2):155-69.

5. Cai B, Liao K, Song XQ, Wei WY, Zhuang Y, Zhang S. Patients with chronically diseased livers have lower incidence of colorectal liver metastases: a meta-analysis. PLoS One 2014;9(9):e108618.

6. Augustin G, Bruketa T, Korolija D, Milosevic M. Lower incidence of hepatic metastases of colorectal cancer in patients with chronic liver diseases: meta-analysis. Hepatogastroenterology 2013;60(125): 1164-8.

7. van Aerts RMM, van de Laarschot LFM, Banales JM, Drenth JPH. Clinical management of polycystic liver disease. J Hepatol 2017 Nov 24. pii: S0168-8278(17)32449-2. Doi: 10.1016/j.hep.2017.11.024. [Epub ahead of print]

8. Wong MY, McCaughan GW, Strasser SI. An update on the pathophysiology and management of polycystic liver disease. Expert Rev Gastroenterol Hepatol 2017;11(6):569-81.

9. Levi Sandri GB, Lai Q, Melandro F, Guglielmo N, Garofalo M, Morabito $\mathrm{V}$, et al. Hepatic resection for giant haemangioma in a patient with a contemporaneous adult polycystic liver disease. Clin Ter 2012;163(5):401-2.

10. lida T, Maeda T, Amari Y, Yurugi T, Tsukamoto Y, Nakajima F. Primary hepatic leiomyosarcoma in a patient with autosomal dominant polycystic kidney disease. CEN Case Rep 2017;6(1):74-8.

11. Malik AK, Filobbos R, Manoharan A, Harvey N, O'Reilly DA, de Liguori CN. A case report of an angiomyxoma in the liver. Ann $R$ Coll Surg Engl 2018;100(4):e81-e84.

12. Koutsampasopoulos $\mathrm{K}$, Antoniadou E, Zoutis S, lacovidis G, Burova 0 , Taplidis A. Neuroendocrine tumor in the liver of a patient with isolated polycystic liver disease: A case report and review of the literature. Oncol Lett 2013;5(5):1664-6.

13. Bhamidimarri KR, Lanza DC, Kashikar ND. Hepatobiliary and Pancreatic: A unique case of HPV related metastatic Schneiderian carcinoma presenting as polycystic liver disease. J Gastroenterol Hepatol 2017;32(1):10.

14. Schnelldorfer T, Torres VE, Zakaria S, Rosen CB, Nagorney DM. Polycystic liver disease: a critical appraisal of hepatic resection, cyst fenestration, and liver transplantation. Ann Surg 2009; 250(1):112-8.

15. Hepatic metastases in a patient with gigantism. Am J Med 1978; 64(2):327-35.

16. Botea F, Nicolaescu D, Barcu A, Picu N, Onofrei A, Droc G, et al. Intraoperative ultrasound guided liver resections: a single center experience. Surg Gastroenterol Oncol 2018;23(1):31-50.

17. Torzilli G, Botea F, Donadon M, Cimino M, Procopio F, Pedicini V, et al. Criteria for the selective use of contrast-enhanced intra-operative ultrasound during surgery for colorectal liver metastases. HPB (Oxford) 2014;16(11):994-1001. 